\title{
Aggressive Staphylococcus lugdunensis Endocarditis in a Young Healthy Patient: A Case Report
}

\author{
Steven Douedi ${ }^{\mathrm{a}}$,, Vandan D. Upadhyaya ${ }^{\mathrm{a}}$, Aref Obagi ${ }^{\mathrm{b}}$, Mohammad Hossain ${ }^{\mathrm{a}}$
}

\begin{abstract}
Staphylococcus lugdunensis ( $S$. lugdunensis) is a $\beta$-hemolytic coagulase-negative staphylococcus causing skin and soft tissue infections with an increasing incidence. Commonly found as normal flora in the perineal region, $S$. lugdunensis has been found in rare cases of infective endocarditis causing increased morbidity and mortality. We present a case of a previously healthy young male diagnosed with $S$. lugdunensis-caused infective endocarditis. A 31-year-old male with no significant past medical history, who presented to the emergency department with acute onset crushing substernal chest pressure and dyspnea with profuse sweating following 1-week-long febrile illness and malaise. The initial electrocardiogram (ECG) showed diffuse ST depressions in all precordial leads, consistent with an acute coronary syndrome. Emergent transthoracic echocardiogram revealed an ejection fraction (EF) of $45 \%$ with severe aortic insufficiency with emanating from a torn right coronary cusp. The patient had a cardiac arrest, and recovery of spontaneous circulation (ROSC) was obtained after $13 \mathrm{~min}$. He subsequently underwent mechanical aortic valve replacement surgery. The native valve specimen cultures grew $S$. lugdunensis. Postoperatively and after a long course of antibiotics, the patient fully recovered without complications. S. lugdunensis is a common organism with increasing incidence that can lead to significant morbidity and mortality if not properly detected and treated. We hope this case presentation would support emergency valve replacement surgery in patients with $S$. lugdunensis-suspected infective endocarditis.
\end{abstract}

Keywords: Staphylococcus lugdunensis; Infective endocarditis; Aortic valve surgery

\section{Introduction}

Staphylococcus lugdunensis (S. lugdunensis) is a gram-posi-

Manuscript submitted February 17, 2020, accepted February 26, 2020

${ }^{a}$ Department of Medicine, Jersey Shore University Medical Center, Hackensack Meridian Health, Neptune, NJ 07753, USA

bepartment of Cardiology, Jersey Shore University Medical Center, Hackensack Meridian Health, Neptune, NJ 07753, USA

${ }^{\mathrm{c}}$ Corresponding Author: Steven Douedi, Internal Medicine Residency Program, Department of Medicine, Jersey Shore University Medical Center, Hackensack Meridian Health, Neptune, NJ 07753, USA.

Email: Steven.Douedi@hackensackmeridian.org

doi: https://doi.org/10.14740/cr1037 tive, coagulase-negative organism [1]. Commonly this organism is present on normal skin flora mostly found in the perineal region [2, 3]. S. lugdunensis can cause bacteremia leading to a significant increase in morbidity and mortality. In rare cases, $S$. lugdunensis has been reported to cause infective endocarditis (IE), producing a large vegetation more commonly in the left heart [4]. We report a case of a young male who was previously healthy with no risk factors presenting with cardiogenic shock and arrest due to $S$. lugdunensis endocarditis requiring emergent surgery.

\section{Case Report}

A 31-year-old male with no significant past medical history who presented from an outlier hospital to our emergency department with an acute onset crushing substernal chest pressure and dyspnea with profuse sweating following 1-week-long febrile illness with malaise. He denied any intravenous drug use, and urine drug screen on admission was negative for illicit substance use. Initial physical exam revealed normal vital signs and clear lungs, but shortly thereafter, the patient rapidly developed dyspnea, tachypnea, and tachycardia. On vital signs patient was noted to be suddenly hypotensive and hypoxic. Neck exam exhibited elevated jugular venous distention and lungs on auscultation revealed significant crackles in bilateral lung fields. The initial electrocardiogram (ECG) showed diffuse ST depressions in all precordial leads, consistent with an acute coronary syndrome (Fig. 1). Thereafter, he underwent an emergent transthoracic echocardiogram showed ejection fraction (EF) of $45 \%$ with severe aortic insufficiency with emanating from a torn right coronary cusp, and cardiothoracic surgery consultation was procured (Fig. 2). Blood cultures came back positive for $S$. lugdunensis in both bottles.

Diagnosis of cardiogenic shock and flash pulmonary edema was suspected, and patient underwent rapid sequence intubation. With the consent of his family, the patient then underwent emergent transesophageal echocardiogram while intubated. This revealed a large vegetation prolapsing into the left ventricular outflow tract, confirming the diagnosis of acute severe aortic insufficiency from endocarditis (Fig. 2). While deliberating with the family about emergent aortic valve replacement, the patient suffered cardiac arrest. Advanced cardiac life support (ACLS) protocol was followed for $13 \mathrm{~min}$, return of spontaneous circulation was restored and the patient was sent for emergent mechanical aortic valve replacement surgery. The native valve specimen cultures grew $S$. lugdunensis. The 


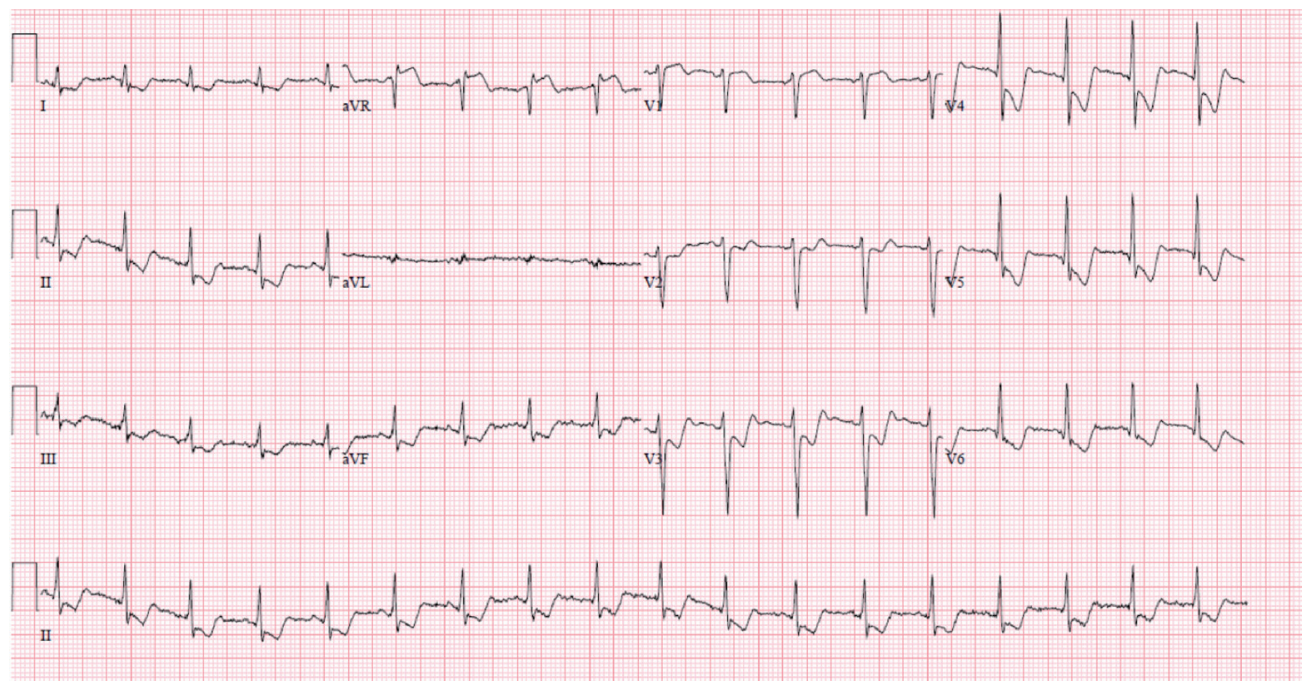

Figure 1. Electrocardiogram showing diffuse ST depression 3 - $4 \mathrm{~mm}$ in all leads except for aVR, which has minimal elevation.

patient had a remarkably uncomplicated postoperative course and was extubated postoperative day 4 , and he was neurologically intact. He was discharged home on postoperative day 8 on intravenous nafcillin to complete a 6 -week course and made a full recovery without disability.

\section{Discussion}

S. lugdunensis is a $\beta$-hemolytic coagulase-negative staphylococcus found to be the cause of about 53 skin and soft tissue infections per 100,000 with an increasing incidence [5]. While part of the normal flora, commonly in the perineal region, it can be aggressive and fatal, when found in the blood, and IE should be thoroughly ruled out $[2,3]$. This organism has also been associated with fatal myocarditis, osteomyelitis, and central nervous system infections leading to cerebrovascular accidents $[6,7]$. The typical valvular pathology involving $S$. lugdunensis usually results in bulky leaflet vegetation usually involving the left-sided heart valves [4]. In a systematic review study, $94.7 \%$ of all IE cases due to this organism were left-sided. Of those, the mitral valve was most affected accounting for $52.5 \%$ of the cases reported, and the aortic valve was documented in $37 \%$ [8]. Our patient presented in this case had aortic valve involvement requiring valve replacement and a 6-week course of intravenous nafcillin. While susceptible to a large range of antibiotics compared to other coagulasenegative staphylococci, $S$. lugdunensis has been found to be resistant to penicillin due to $\beta$-lactamase production [9]. Taha et al reported penicillin resistance to be up to $45 \%$ in the USA and even high in other countries [9-11]. Resistance to methicillin antibiotics however have been far less common due to lack

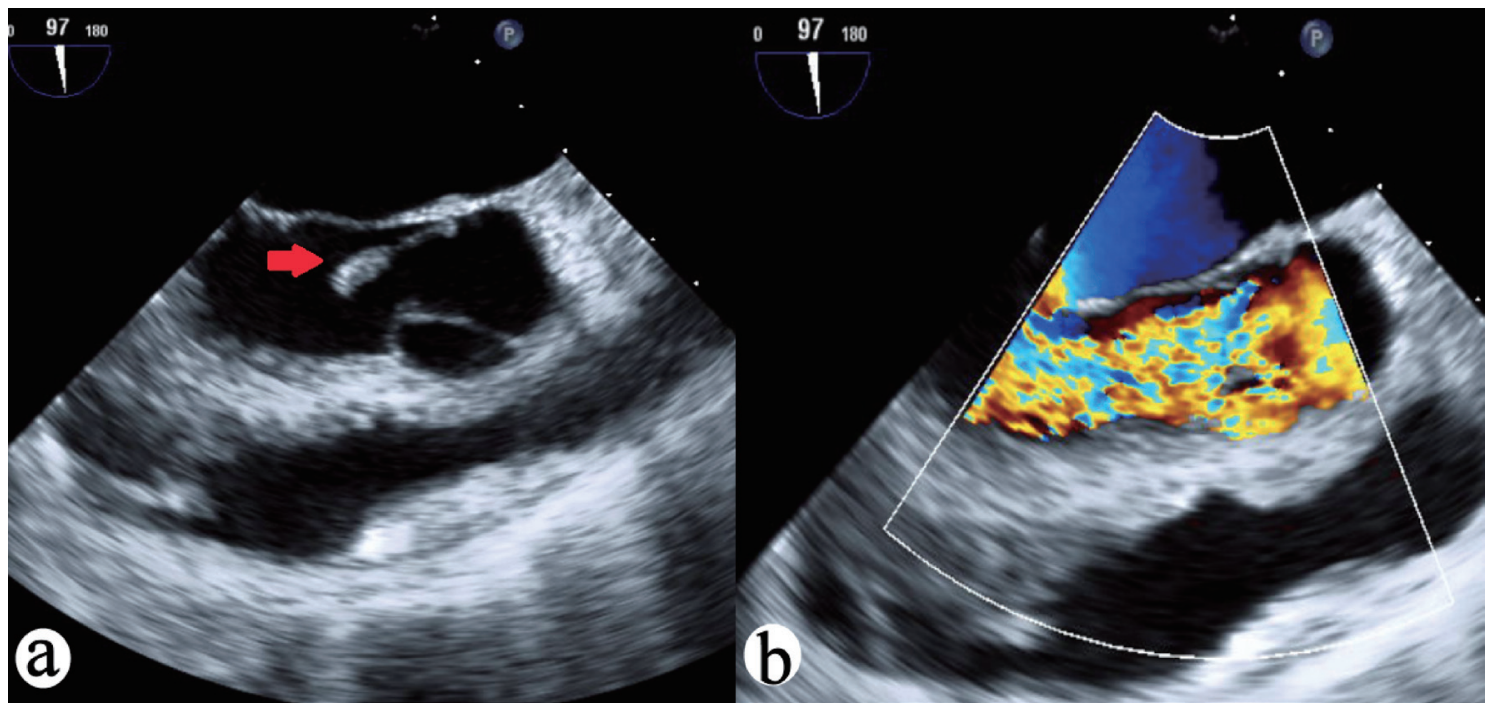

Figure 2. (a) TEE 97-degree view of aortic valve vegetation (red arrow) with prolapse. (b) TEE 97-degree view of aortic valve with wide open aortic regurgitation. TEE: transesophageal echocardiography. 
of the mec $A$ gene $[9,12]$. For this reason and due to the patient presenting with IE, intravenous nafcillin was the antibiotic of choice for our patient. Although rare in the USA, the emergence of methicillin antibiotic resistance has been documented in literature [9]. In an epidemiological study, Yen et al reported an $87 \%$ penicillin resistance and $20 \%$ methicillin resistance in $S$. lugdunensis found to have the mecA gene [11]. Other alternatives for treatment include clindamycin and erythromycin, both of which have been found to have low resistance prevalence $[9,10]$.

Because the organism binds directly to von Willebrand factor (VWF), it adheres to the valves and vessels firmly [13]. The organism is very resistant to shear forces, explaining its aggressiveness compared to other coagulase-negative staphylococci [13]. This mechanism of attachment is also seen in Staphylococcus aureus and other aggressive bacteria. This makes $S$. lugdunensis extremely difficult to treat and poses a high morbidity and mortality. Patients with $S$. lugdunensis endocarditis are more likely to present with severe and rapid onset symptoms of heart failure and decreased organ perfusion leading to shock, as also seen in our patient [14]. In a prospective cohort study, Anguera et al reported a mortality rate of up to $42 \%$ in native valves and up to $78 \%$ in prosthetic valves [4]. Despite such poor outcomes, early surgical management of $S$. lugdunensis IE is recommended along with an aggressive intravenous antibiotic regimen $[15,16]$, and has been shown to significant decrease mortality in these patients [4]. In our case, rapid identification and early surgical treatment proved to be lifesaving.

\section{Conclusions}

S. lugdunensis is a common organism with increasing incidence, which can lead to significant morbidity and mortality if not properly detected and treated. Early surgical intervention has been shown to significantly improve patient outcomes. We hope our report of a near fatal case of S. lugdunensis endocarditis in a young, previously healthy patient successfully treated with emergency valve replacement surgery supports that the use of heroic supportive treatment measures as being effective and warranted in viable patients.

\section{Acknowledgments}

None to declare.

\section{Financial Disclosure}

The authors did not obtain funding for the publication of the manuscript.

\section{Conflict of Interest}

The authors declare that there is no conflict of interest regard- ing the publication of this paper.

\section{Informed Consent}

The patient described in the case report had given informed consent for the case report to be published.

\section{Author Contributions}

Each author has individually been involved in and has made substantial contributions to case selection, case presentation, discussion, drafting manuscript and revision and final approval.

\section{Data Availability}

All data and information regarding this manuscript are present within the text.

\section{References}

1. Heldt Manica LA, Cohen PR. Staphylococcus lugdunensis Infections of the Skin and Soft Tissue: A Case Series and Review. Dermatol Ther (Heidelb). 2017;7(4):555562.

2. Bellamy R, Barkham T. Staphylococcus lugdunensis infection sites: predominance of abscesses in the pelvic girdle region. Clin Infect Dis. 2002;35(3):E32-34.

3. van der Mee-Marquet N, Achard A, Mereghetti L, Danton A, Minier M, Quentin R. Staphylococcus lugdunensis infections: high frequency of inguinal area carriage. J Clin Microbiol. 2003;41(4):1404-1409.

4. Anguera I, Del Rio A, Miro JM, Matinez-Lacasa X, Marco F, Guma JR, Quaglio G, et al. Staphylococcus lugdunensis infective endocarditis: description of 10 cases and analysis of native valve, prosthetic valve, and pacemaker lead endocarditis clinical profiles. Heart. 2005;91(2):e10.

5. Bocher S, Tonning B, Skov RL, Prag J. Staphylococcus lugdunensis, a common cause of skin and soft tissue infections in the community. J Clin Microbiol. 2009;47(4):946-950.

6. Kuzhively J, Patel SA, Abraham H. The Long CoN(S): A case of staphylococcus lugdunensis endocarditis with cerebral and coronary embolism. J Med Cases. 2014;5:535537.

7. Ngan ATL, Lee S, Liu T, Tam M, Li KHC, Wong MV, Lam MHS, et al. A rare case of Staphylococcus lugdunensis septicemia associated with myocarditis and atrioventricular block. J Geriatr Cardiol. 2019;16(1):63-66.

8. Kyaw H, Raju F, Shaikh AZ, Lin AN, Lin AT, Abboud J, Reddy S. Staphylococcus lugdunensis endocarditis and cerebrovascular accident: a systemic review of risk factors and clinical outcome. Cureus. 2018;10(4):e2469.

9. Taha L, Stegger M, Soderquist B. Staphylococcus lug- 
dunensis: antimicrobial susceptibility and optimal treatment options. Eur J Clin Microbiol Infect Dis. 2019;38(8):1449-1455.

10. Kleiner E, Monk AB, Archer GL, Forbes BA. Clinical significance of Staphylococcus lugdunensis isolated from routine cultures. Clin Infect Dis. 2010;51(7):801-803.

11. Yen TY, Sung YJ, Lin HC, Peng CT, Tien N, Hwang KP, $\mathrm{Lu}$ JJ. Emergence of oxacillin-resistant Staphylococcus lugdunensis carrying staphylococcal cassette chromosome mec type $\mathrm{V}$ in central Taiwan. J Microbiol Immunol Infect. 2016;49(6):885-891.

12. Ishiekwene $\mathrm{C}$, Ghitan M, Kuhn-Basti M, Chapnick E, Lin YS. Staphylococcus lugdunensis endocarditis with destruction of the ventricular septum and multiple native valves. IDCases. 2017;7:14-15.

13. Nilsson M, Bjerketorp J, Wiebensjo A, Ljungh A,
Frykberg L, Guss B. A von Willebrand factor-binding protein from Staphylococcus lugdunensis. FEMS Microbiol Lett. 2004;234(1):155-161.

14. Viganego F, Nazir TM, Lee CY, Hafner JP, Smith R. Staphylococcus lugdunensis endocarditis following lower extremity revascularization. Int J Cardiol. 2007;116(1):e46.

15. Van Hoovels L, De Munter P, Colaert J, Surmont I, Van Wijngaerden E, Peetermans WE, Verhaegen J. Three cases of destructive native valve endocarditis caused by Staphylococcus lugdunensis. Eur J Clin Microbiol Infect Dis. 2005;24(2):149-152.

16. Takahashi N, Shimada T, Ishibashi Y, Yoshitomi H, Sugamori T, Sakane T, Sato H, et al. The pitfall of coagulasenegative staphylococci: a case of Staphylococcus lugdunensis endocarditis. Int J Cardiol. 2009;137(1):e15-17. 